\title{
Analysis of $L K B 1$ mutations and other molecular alterations in pancreatic acinar cell carcinoma
}

\author{
Roeland F de Wilde ${ }^{1,2}$, Niki A Ottenhof ${ }^{1}$, Marnix Jansen ${ }^{3}$, Folkert HM Morsink ${ }^{1}$, \\ Wendy WJ de Leng ${ }^{1}$, G Johan A Offerhaus ${ }^{1,3}$ and Lodewijk AA Brosens ${ }^{1}$ \\ ${ }^{1}$ Department of Pathology, University Medical Center Utrecht, Utrecht, The Netherlands; ${ }^{2}$ Department of \\ Surgery, University Medical Center Utrecht, Utrecht, The Netherlands and ${ }^{3}$ Department of Pathology, \\ Academic Medical Center, Amsterdam, The Netherlands
}

\begin{abstract}
Acinar cell carcinoma is a rare non-ductal neoplasm of the pancreas with poorly defined molecular genetic features. Recently, biallelic inactivation of $L K B 1$ was described in an acinar cell carcinoma of a Peutz-Jeghers patient carrying a heterozygous germline $L K B 1$ mutation, and inhibition of $\mathrm{mTOR}$ signaling resulted in partial remission of the tumor. To explore the potential of mTOR inhibitors in sporadic acinar cell carcinoma, the $L K B 1$ gene was investigated in five sporadic acinar cell carcinomas by sequence analysis, methylation analysis and mRNA expression. In addition, microsatellite instability and methylation of a number of tumor suppressor genes were investigated and KRAS, TP53, CDKN1A, SMAD4 and CTNNB1 were studied by mutation analysis and immunohistochemistry. No mutations, deletions or promoter hypermethylation of LKB1 were found in any of the sporadic acinar cell carcinomas, and mRNA expression of LKB1 was not altered. Amplifications at chromosome $20 q$ and $19 p$ were found in 100 and $80 \%$ of the cases, respectively. In addition, hypermethylation of one or more tumor suppressor genes was found in $80 \%$ of cases. One case harbored a TP53 mutation, and expression of SMAD4 and CTNNB1 was altered in one case each. No KRAS mutations or microsatellite instability were found. To conclude, no evidence for a role for LKB1 in tumorigenesis of sporadic pancreatic acinar cell carcinoma was found. However, copy number variations and hypermethylation were found in a majority of cases. Molecular pathways involved in acinar cell carcinoma-tumorigenesis differ from those involved in ductal pancreatic neoplasms. Further studies are needed to increase our understanding of molecular pathogenesis of acinar cell carcinoma, which may eventually result in development of new therapeutic targets.

Modern Pathology (2011) 24, 1229-1236; doi:10.1038/modpathol.2011.83; published online 13 May 2011
\end{abstract}

Keywords: acinar cell carcinoma; copy number variation; LKB1/STK11; methylation; mTOR; pancreas; Peutz-Jeghers syndrome

Acinar cell carcinoma is a rare, non-ductal neoplasm of the exocrine pancreas derived from acinar cells. It represents about $1-2 \%$ of all exocrine pancreatic neoplasms. ${ }^{1}$ The mean age at diagnosis is 62 years, although several pediatric cases have been described. ${ }^{2-5}$ The vast majority $(85-90 \%)$ of pancreatic exocrine neoplasms are pancreatic ductal

Correspondence: Dr LAA Brosens, MD, Department of Pathology, University Medical Center Utrecht, H04.312, Heidelberglaan 100, Utrecht 3584CX, The Netherlands.

E-mail: l.a.a.brosens@umcutrecht.nl

Received 28 February 2011; revised 6 April 2011; accepted 6 April 2011; published online 13 May 2011 adenocarcinomas, for which several morphologically distinct precursor lesions have been identified, including pancreatic intraepithelial neoplasia, intraductal papillary mucinous neoplasm, and mucinous cystic neoplasm. ${ }^{6}$ With a mean survival of 18 months and a 5-year survival rate of less than $10 \%$, the prognosis of acinar cell carcinoma is slightly better than that of pancreatic ductal adenocarcinoma. $^{2,3}$

Histologically, acinar cell carcinomas are often well circumscribed and sometimes multinodular lesions composed of large nodules of cells separated by hypocellular fibrous bands. A desmoplastic stroma reaction, characteristic for pancreatic ductal 
adenocarcinoma, is usually lacking. Neoplastic cells are characterized by a minimal to moderate amount of granular cytoplasm and round to oval uniform nuclei..$^{2,3,5}$ Identification of pancreatic enzymes by immunohistochemistry, such as trypsin, chymotrypsin and lipase can be helpful in establishing the diagnosis. ${ }^{2,3}$ In some patients, hypersecretion of lipase by the neoplasm can produce a distinctive syndrome of subcutaneous fat necrosis and polyarthralgia. Molecular features of pancreatic acinar cell carcinoma have not been well characterized, but, in contrast to pancreatic ductal adenocarcinoma, acinar cell carcinomas rarely show KRAS, TP53, or SMAD4 mutations. ${ }^{2,7-12}$ APC or CTNNB1 mutations have been found in a subset (23.5\%) of acinar cell carcinomas. ${ }^{10}$

Recently, acinar cell carcinoma of the pancreas was described for the first time in a patient with Peutz-Jeghers syndrome and biallelic inactivation of $L K B 1$ allele was demonstrated in this tumor, indicating a causative role for $L K B 1$ in the development of this neoplasm. Moreover, treatment with an mammalian target of rapamycin (mTOR) inhibitor resulted in partial remission of the tumor. ${ }^{13}$

Peutz-Jeghers syndrome is a rare autosomal dominant disorder caused by germline mutation of LKB1 and characterized by mucocutaneous melanin pigmentation and distinctive non-neoplastic hamartomatous intestinal polyps. ${ }^{14}$ Peutz-Jeghers syndrome patients are at increased risk for gastrointestinal and non-gastrointestinal malignancies, including pancreatic cancer. The relative risk of pancreatic cancer in Peutz-Jeghers syndrome has been estimated to be as high as 132 and the lifetime risk $36 \% .{ }^{15}$ Reported cases of pancreatic cancer in Peutz-Jeghers syndrome mainly involve pancreatic ductal adenocarcinoma and mucinous cystadenocarcinoma, but serous cystadenoma, intraductal papillary mucinous neoplasms, and papillary adenoma of the pancreatic duct have also been reported. ${ }^{16-19}$ Moreover, $L K B 1$ has previously been shown to have a role in the pathogenesis of intraductal papillary mucinous neoplasms, sporadic pancreatic, and biliary carcinomas. ${ }^{17,20}$

$L K B 1$ is involved in the establishment of cell polarity and in the regulation of cellular response to energy and stress. Specifically, LKB1 activates adenosine monophosphate-activated protein kinase (AMPK) among other kinases, and leads to inhibition of mTOR. mTOR is a highly conserved serine/ threonine kinase that regulates protein synthesis and cell growth. Loss of $L K B 1$ function, therefore, leads to increased mTOR signaling and increased cell growth and is a treatment-specific target of interest. ${ }^{14}$

In view of the recent report of an acinar cell carcinoma with second hit inactivation of $L K B 1$ in a Peutz-Jeghers syndrome patient, our aim was to further investigate molecular alterations in acinar cell carcinomas and, specifically, to investigate whether $L K B 1$ is involved in acinar cell carcinoma carcinogenesis.

\section{Methods}

\section{Patient Samples and DNA Isolation}

Archival formalin-fixed paraffin-embedded tissue was obtained from the Peutz-Jeghers syndrome patient (PJS1) described by Klümpen et $a l,{ }^{13}$ and five confirmed sporadic pancreatic acinar cell carcinoma cases (T1-5) from the surgical pathology archives of the Academic Medical Center Amsterdam $(n=3)$, Onze Lieve Vrouwe Gasthuis Amsterdam $(n=1)$ and University Medical Center Utrecht $(n=1)$. A panel of immunohistochemical markers, including vimentin, keratin markers, trypsin, chymotrypsin, lipase, CD10, CD56, synaptofysin, chromogranin and NSE, was used in establishing the diagnosis (data not shown). The study was carried out according to the guidelines and with approval of the ethics committee of these institutions.

Formalin-fixed paraffin-embedded tissue sections were used for immunohistochemistry analysis and genomic DNA isolation. DNA was isolated after deparaffinization using the QIAamp DNA mini Kit (Qiagen). DNA concentrations were determined using the NanoDrop 2000 Spectrophotometer (Thermo Scientific).

Frozen tumor tissue was available from three cases (T2-4) and corresponding normal pancreatic tissue of cases $\mathrm{T} 2$ and $\mathrm{T} 3$ (T2 NP1/NP2 and T3_NP1). In addition, five independent normal frozen pancreatic tissue specimens (C1-5) were available from the tissue bank of the University Medical Center Utrecht.

\section{Mutation Analysis}

$L K B 1$ mutation analysis was carried out in all tumor samples using primers for all coding exons and exon/intron boundaries of the $L K B 1$ gene, as previously described. ${ }^{21}$ Sequence analysis of exons 1 and 2 of KRAS and exons 4-9 of TP53 was carried out in all tumor samples. Primer sequences and PCR conditions are available on request.

After purification, the sequence reaction was carried out using the Big Dye Terminator Cycle Sequencing Kit (Applied Biosystems, Foster City, CA, USA). Samples were run on a 3730 genetic analyzer (Applied Biosystems) and analyzed using CodonCode Aligner software (CodonCode Corporation, Dedham, MA, USA).

\section{(Methylation Specific) Multiplex Ligation-dependent Probe Amplification ((ms-)MLPA)}

Copy number variations in the $L K B 1$ gene were studied using the MLPA kit P101 (MRC-Holland, Amsterdam, The Netherlands) as previously described. ${ }^{21}$ Four genomic DNA samples derived from peripheral blood from healthy individuals 
served as normal controls, to which control probes and samples were normalized. Deletions and duplications were defined as ratios of $<0.5$ and $>1.75$, respectively, and were repeated at least two times. ${ }^{21}$

CpG island promoter methylation status of $L K B 1$ and 24 other tumor suppressor (including mTOR pathway genes PTEN, TSC2, and VHL) was investigated using the ms-MLPA ME002-B1 tumor suppressor kit (MRC-Holland), according to manufacturer's protocol. Two negative control samples containing unmethylated DNA and one positive control containing completely methylated DNA were analyzed simultaneously. Samples were run on an ABI 3730 genetic analyzer and results were analyzed using the MRC Coffalyser software (MRC-Holland). Methylation was defined as a percentage higher than $25 \%$. All samples were analyzed in duplicate.

\section{LKB1 mRNA Expression}

Quantitative analysis of $L K B 1$ mRNA expression levels was assessed in three of the acinar cell carcinomas, of which frozen tissue was available (cases T2-4). Corresponding normal pancreatic tissue of two acinar cell carcinoma cases (T3_NP1/ NP2 and T4_NP1) and five normal pancreata (C1-5) were included to serve as normal controls. The housekeeping gene GAPDH was used as an internal control for reverse transcription in all samples. mRNA was isolated using Trizol reagent (Invitrogen, Carlsbad, CA, USA) according to the manufacturer's protocol. cDNA was prepared from $1 \mu \mathrm{g}$ mRNA using Superscript III (Invitrogen) and a combination of random hexamers and oligo-(dT) primers (Invitrogen). RT-PCR for $L K B 1$ and $G A P D H$ was performed using an Assay on Demand (LKB1: Hs00176092_m1, GAPDH: Hs99999905_m1, Applied Biosystems) according to the manufacturers protocol in the ABI 7900 (Applied Biosystems). Samples were tested in duplicate. Relative expression was determined using SDS2.2.1 software (Applied Biosystems).

\section{Microsatellite Analysis}

DNA was isolated by direct lysis using ProtK after microdissection of tumor and normal formalin-fixed paraffin-embedded tissue of the same patient. Next, tumor and normal DNA were PCR-amplified using six markers recommended by the National Cancer Institute to screen for microsatellite instability in hereditary non-polyposis colorectal cancer (so called Bethesda markers, BAT25, BAT26, BAT40, D17S250, D2S123, and D5S346). ${ }^{22}$

After PCR amplification, $1 \mu \mathrm{l}$ of each product was added to $0.5 \mu \mathrm{l}$ of GS500 size marker and $23.5 \mu \mathrm{l}$ MilliQ. The amplified fragments were separated by denaturing gel electrophoresis using an ABI 3730 genetic analyzer (Applied Biosystems). Data were analyzed using GeneMapper software (Applied Biosystems). Microsatellite instability was defined as the presence of extra or shifted bands in comparison with the normal control. The tissues were classified as microsatellite high instability, if instability was noted in $30 \%$ of the loci investigated, microsatellite low instability, if instability was observed in $<30 \%$ of the loci investigated, and microsatellite stable, if there was no instability. Primer sequences and PCR conditions are available on request.

\section{Immunohistochemistry}

Immunohistochemistry on all formalin-fixed paraffin-embedded tissue samples for CTNNB1, TP53, and SMAD4 was performed and scored as described before. $^{23}$ CDKN1A immunohistochemistry was performed using a monoclonal antibody (Invitrogen, $18-0401 ; 1: 150)$ according to the TP53/SMAD4 protocol and results were scored with respect to intensity (negative, weak, or positive). Immunohistochemistry of mismatch repair proteins MSH6, MSH2, MLH1, PMS2 was performed in the Leica Bond III fully automated immunohistochemistry stainer. Detailed conditions are available on request. All slides were scored by an experienced GI pathologist (GJAO).

\section{Results}

\section{LKB1 Sequence Analysis and MLPA}

All nine coding exons and exon/intron boundaries of the $L K B 1$ gene were analyzed by sequence analysis and MLPA in all five acinar cell carcinoma

Table 1 Summary of copy number and methylation analysis by (methylation specific) MLPA

\begin{tabular}{|c|c|c|c|c|c|c|c|c|}
\hline \multirow{2}{*}{$\begin{array}{l}\text { Case } \\
\mathrm{T} 1\end{array}$} & \multirow{2}{*}{$\begin{array}{l}\text { Age/sex } \\
\text { M/48 }\end{array}$} & \multicolumn{2}{|c|}{ Copy number analysis: amplification gene (chromosomal position) } & \multicolumn{5}{|c|}{ Methylation } \\
\hline & & GATA5 (20q13.3) & $L K B 1^{\mathrm{a}}(19 p 13.3)$ & ESR1 & MSH6 & & THBS1 & $\mathrm{CDH} 13$ \\
\hline $\mathrm{T} 2$ & $\mathrm{~F} / 68$ & GATA5 (20q13.3) & $L K B 1^{\mathrm{a}}(19 p 13.3)$ & ESR1 & & & GSTP1 & \\
\hline T3 & $\mathrm{M} / 61$ & GATA5 (20q13.3) & & & & & & \\
\hline $\mathrm{T} 4$ & $\mathrm{~F} / 19$ & GATA5 (20q13.3) & BRCA2 (13q13.1) & ESR1 & & PAX5 & PAX6 & WT1 \\
\hline T5 & $\mathrm{M} / 33$ & GATA5 (20q13.3) & $L K B 1^{\mathrm{a}}(19 p 13.3)$ & & MSH6 & PAX5 & $C D 44$ & \\
\hline Total & & $5 / 5$ & $4 / 5$ & $3 / 5$ & $2 / 5$ & $2 / 5$ & & \\
\hline
\end{tabular}

Abbreviations: M, male; F, female.

${ }^{\mathrm{a} C h r o m o s o m e} 19$ amplification.

${ }^{\mathrm{b}}$ Amplification of two probes. 


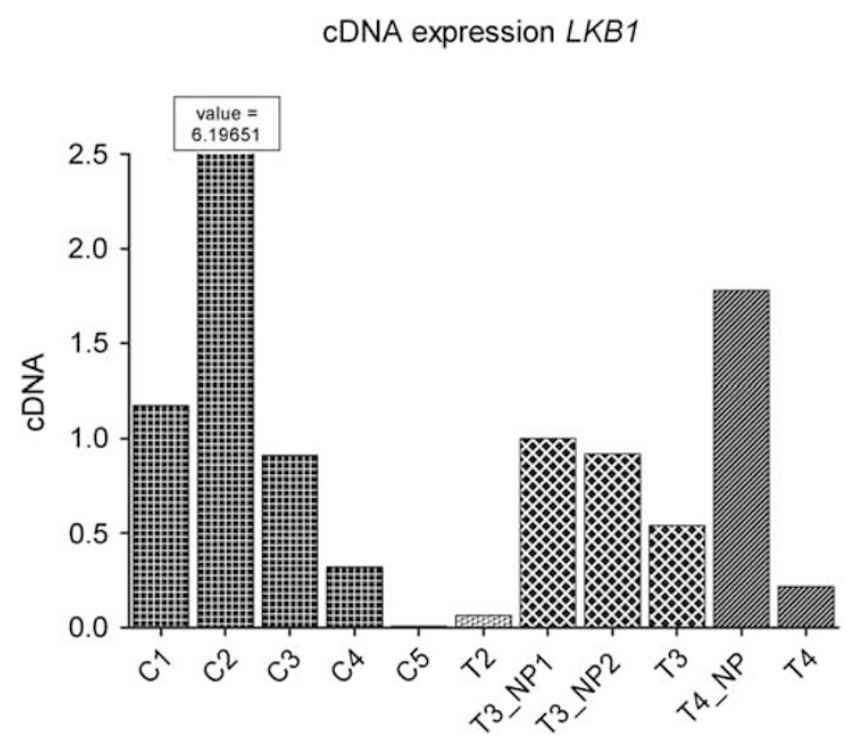

Figure 1 Relative $L K B 1$ cDNA expression levels in five normal control pancreata (C1-5) and three pancreatic acinar cell carcinomas (T2-4). NP: normal pancreas.

cases (T1-5). Sequence analysis did not reveal any alterations in $L K B 1$ and MLPA analysis did not show deletions in the $L K B 1$ gene. Amplification of $L K B 1$ was found in four cases, including three samples (T1, T2 and T5) with complete amplification of chromosome 19 containing LKB1. GATA5 amplification was found in all five cases. BRCA2 and $R B 1$ were amplified in one case (Table 1).

\section{Methylation-Specific MLPA}

CpG island promoter methylation of $L K B 1$ and 24 other tumor suppressor genes was studied using ms-MLPA in all five acinar cell carcinoma cases (T1-5) (Table 1). No hypermethylation of the LKB1 promoter was found in any of the samples. However, three tumors showed hypermethylation of the ESR1 promoter and two showed hypermethylation of $M S H 6$ and PAX5. CD44, CDH13, GSTP1, PAX6, THBS1, and WT1 were hypermethylated in one tumor.

\section{LKB1 Quantitative mRNA Analysis}

Quantitative mRNA analysis did not show significant differences in $L K B 1$ mRNA expression between corresponding normal and tumor and normal control samples (Figure 1), which is in line with the results of the mutation and MLPA analysis. Frozen tissue was available from one patient, with chromosome 19 amplification (T2) and one with $L K B 1$ amplification (T4) but did not show aberrant $L K B 1$ mRNA expression.

\section{KRAS and TP53 Mutation Analysis}

Mutation analysis of KRAS did not show any mutations. TP53 mutation analysis revealed two
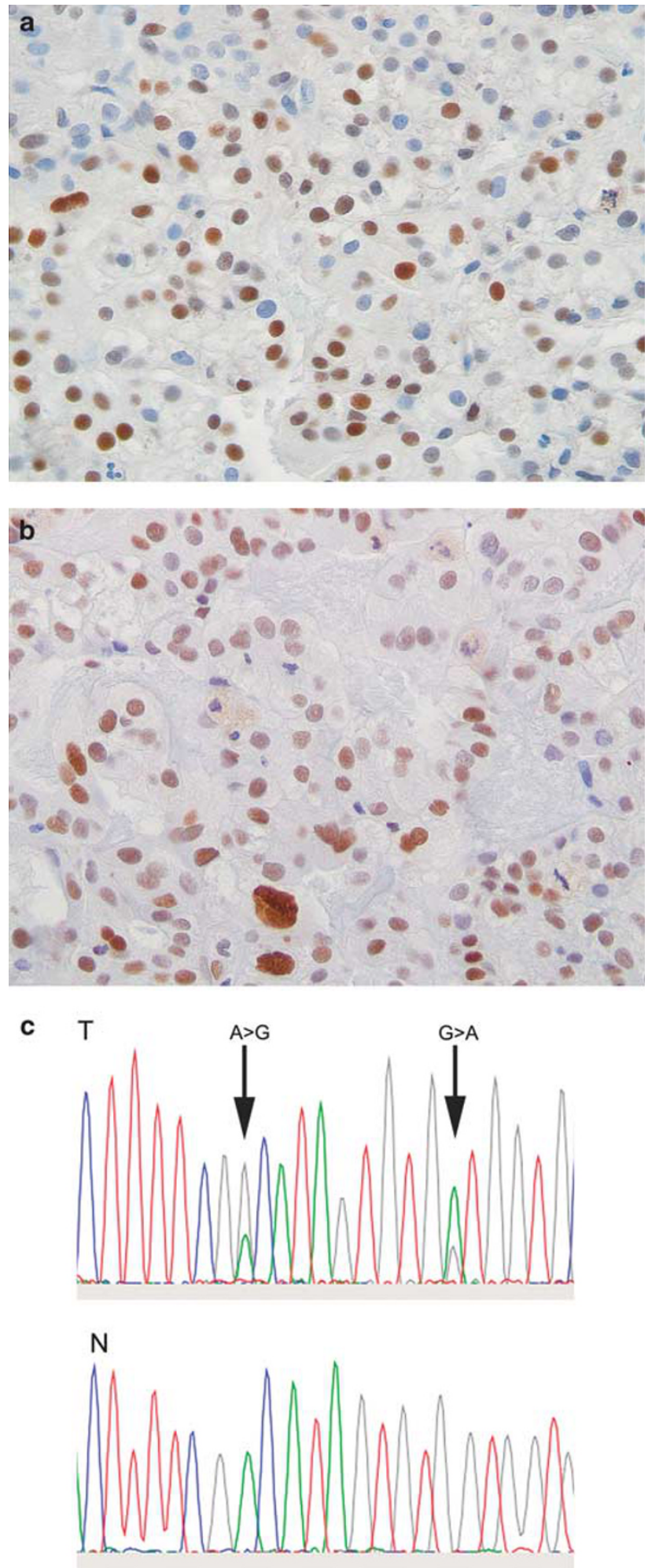

Figure 2 TP53 and CDKN1A immunohistochemistry and TP53 mutation analysis of case T1. (a) This case showed nuclear accumulation of TP53, and (b) positive CDKN1A immunostaining. (c) Sequence analysis of TP53 shows a silent mutation in codon 213 (R213R) and a missense mutation in codon 217 (V217M), which is in accordance with the positive TP53 immunostaining shown in panel a. 
mutations in exon 6 in case T1 (one silent mutation in codon 213 (R213R) and one missense mutation in codon 217 (V217M)) (Figure 2c). KRAS and TP53 mutation analysis could not be completed in case T5 because of insufficient DNA (Table 2).

\section{Immunohistochemistry of TP53, CTNNB1, SMAD4 and CDKN1A}

Results of immunohistochemistry are summarized in Table 2. TP53 immunolabeling showed nuclear accumulation in case $\mathrm{T} 1$, which is in accordance with the mutation that was found in this case (Figures 2a and c). Aberrant expression of CTNNB1 and SMAD4 was found in two different cases (case $\mathrm{T} 2$ and T3, respectively) (Figures $3 \mathrm{a}$ and b). Immunolabeling for CDKN1A showed variable staining intensity from positive to absent (Figures $2 \mathrm{~b}$ and 3c). Interestingly, positive CDKN1A staining was found in the case harboring the TP53 mutation (Figure 2b).

\section{Microsatellite Instability and Immunohistochemistry of Mismatch Repair Genes}

All tumors were microsatellite stable and showed normal expression of the mismatch repair proteins MLH1, MSH2, MSH6, and PMS2. However, one case (PJS1) showed loss of heterozygosity (LOH) of D17S250 and D5S346 and one case (T3) showed LOH of D17S250 (Table 2).

\section{Discussion}

Acinar cell carcinoma is a rare non-ductal neoplasm of the exocrine pancreas and associated with a poor prognosis. Molecular alterations in acinar cell carcinoma carcinogenesis are poorly characterized. Only few studies investigated molecular alterations in a limited number of acinar cell carcinomas (Table 3). These studies do not reveal specific molecular alterations that characterize acinar cell carcinoma carcinogenesis, although it does become clear that genes commonly involved in pancreatic ductal adenocarcinoma such as KRAS, TP53, and SMAD4 do not have key roles in acinar cell carcinoma carcinogenesis. In addition, one study reported Wnt pathway activation in $24 \%$ of acinar cell carcinoma cases and copy number variations have been reported by two investigators. ${ }^{2,7-12,24} \mathrm{CpG}$ island promoter methylation has not been studied in acinar cell carcinoma before.

Recently, acinar cell carcinoma of the pancreas was described for the first time in a patient with Peutz-Jeghers syndrome. ${ }^{13}$ A causal relationship of Peutz-Jeghers syndrome, caused by heterozygous germline mutation of the $L K B 1$ gene, and this acinar cell carcinoma was demonstrated by showing loss of the wild-type $L K B 1$ allele in the tumor. In addition, a partial response of the tumor on treatment with an mTOR inhibitor was shown, which fits with the notion of complete LKB1 inactivation leading to aberrant mTOR signaling in this tumor. ${ }^{14}$ The mTOR inhibitor sirolimus was previously also reported to suppress and prevent polyp growth in murine Peutz-Jeghers syndrome models and tumor growth in a mouse model for acinar cell carcinoma. ${ }^{25-28}$ Moreover, alterations in mTOR signaling are found in a subset $(14 \%)$ of pancreatic neuroendocrine tumors and $L K B 1$ inactivation is found in pancreatic neoplasms of ductal origin. ${ }^{17,20,29}$

On the basis of these observations, our aim was to investigate whether $L K B 1$ inactivation is involved in tumorigenesis of sporadic pancreatic acinar cell carcinomas, and to address the potential role for mTOR inhibitors in treatment of sporadic acinar cell carcinoma. However, we did not find inactivation of $L K B 1$ by somatic mutation, deletion, or promoter hypermethylation in five sporadic acinar cell carcinomas. This was further substantiated by the finding of normal $L K B 1$ mRNA expression levels in three studied cases. Moreover, no methylation or deletions were found in three other mTOR pathway genes (PTEN, TSC2, and VHL) by (ms-)MLPA. Although investigated in a limited number of cases,

Table 2 Summary of KRAS and TP53 mutation analysis, microsatellite analysis and immunohistochemistry

\begin{tabular}{|c|c|c|c|c|c|c|c|c|c|}
\hline \multirow[t]{2}{*}{ Case } & \multirow[t]{2}{*}{ Sex/age } & \multicolumn{2}{|c|}{ Mutation analysis } & \multicolumn{4}{|c|}{ Immunohistochemistry } & \multirow[t]{2}{*}{$M S I$} & \multirow[t]{2}{*}{$\mathrm{LOH}$} \\
\hline & & TP53 & KRAS & TP53 & $C D K N 1 A$ & SMAD4 & CTNNB1 & & \\
\hline PJS1 & $\mathrm{M} / 45$ & WT & WT & - & 0 & + & Membranous & MSS & D17S250, D5S346 \\
\hline $\mathrm{T} 1$ & $\mathrm{M} / 48$ & S \& MS & WT & + & 2 & + & Membranous & MSS & \\
\hline $\mathrm{T} 2$ & $\mathrm{~F} / 68$ & WT & WT & - & 0 & + & Nuclear & MSS & \\
\hline T3 & $\mathrm{M} / 61$ & WT & WT & - & 2 & - & Membranous & MSS & D17S250 \\
\hline $\mathrm{T} 4$ & $\mathrm{~F} / 19$ & WT & WT & - & 0 & + & Membranous & MSS & \\
\hline T5 & $\mathrm{M} / 33$ & $\mathrm{NA}^{\mathrm{a}}$ & $\mathrm{NA}^{\mathrm{a}}$ & - & 1 & + & Membranous & MSS & \\
\hline
\end{tabular}

Abbreviations: M, male; F, female; PJS, Peutz-Jeghers syndrome; WT, wild type; S, silent mutation; MS, missense mutation; MSI, microsatellite instability; MSS, microsatellite stable; LOH, loss of heterozygosity; NA, not applicable.

For TP53-normal negative labeling; +, positive labeling. For CDKN1A: 0, absent labeling; 1, weak positive labeling; 2, strong positive labeling. For SMAD4-absent labeling; +, normal positive labeling. For CTNNB1: N, normal membraneous (negative); F, focal nuclear labeling (positive).

${ }^{\mathrm{a}}$ Insufficiënt DNA. 

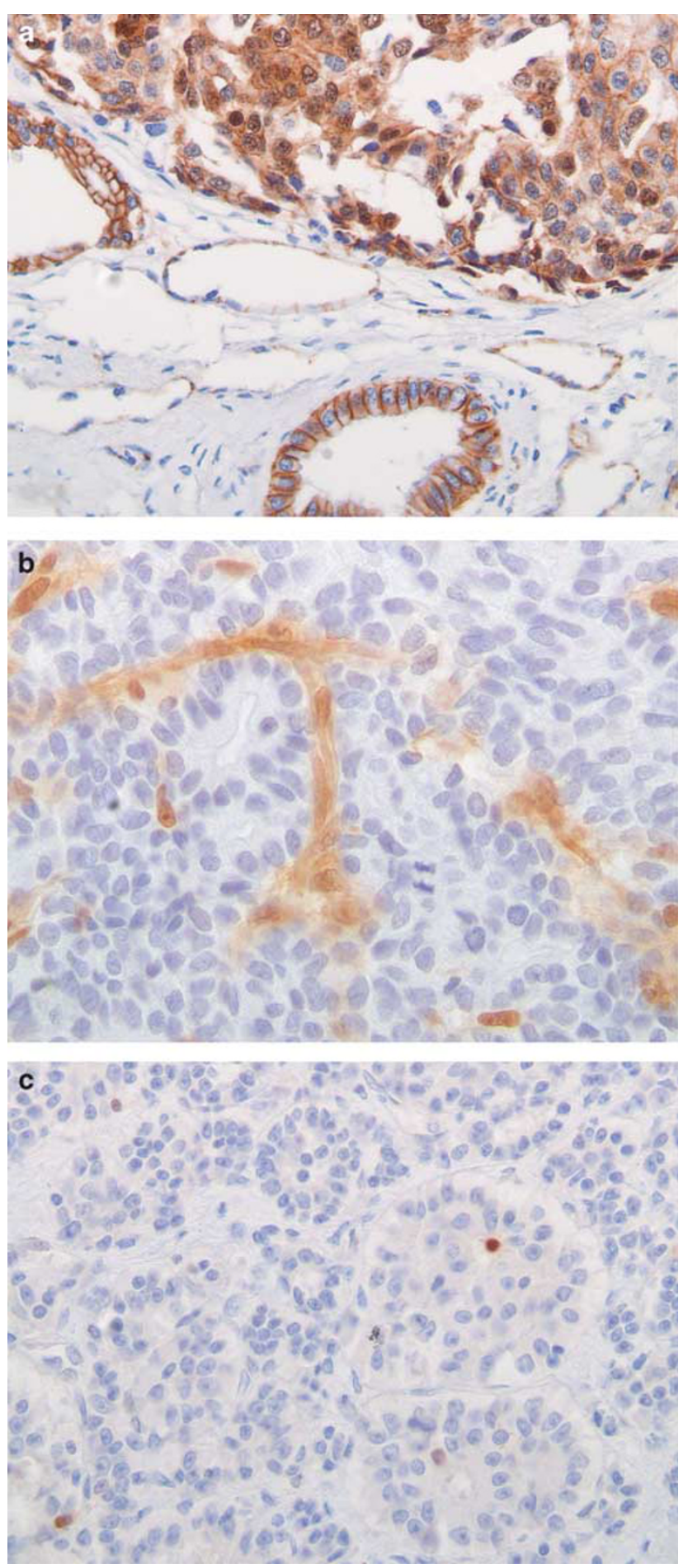

Figure 3 Microphotographs of immunohistochemistry. (a) Nuclear expression of CTNNB1 in an acinar cell carcinoma (T2). (b) Loss of SMAD4 expression in an acinar cell carcinoma (T3). (c) Negative CDKN1A expression in an acinar cell carcinoma (T4).

these results do not point to a role for $L K B 1$ or disturbed mTOR signaling in the tumorigenesis of sporadic acinar cell carcinomas.

Interestingly, studies using conditional knockout mice have shown that targeted deletion of $L k b 1$ and
Table 3 Summary of molecular alterations in pancreatic acinar cell carcinomas reported in the literature

\begin{tabular}{lrll}
\hline Genetic alteration & $\begin{array}{c}\text { Cases } \\
\text { studied }\end{array}$ & $\begin{array}{c}\text { Abnormal } \\
\text { cases (\%) }\end{array}$ & References \\
\hline KRAS & 36 & $1(3 \%)$ & $2,7,8,11$ \\
TP53 & 69 & $0(0 \%)$ & $2,7,9-12$ \\
CDKN1A mutation & 6 & $0(0 \%)$ & 11 \\
SMAD4 & 35 & $0(0 \%)$ & $10-12$ \\
APC/CTNNB1 & 17 & $4^{\mathrm{a}}(24 \%)$ & 10 \\
MSI & 13 & $3^{\mathrm{b}}(23 \%)$ & 10 \\
Gain at 1q, 12p, Xq, & 6 & NA (50-60\%) & 24 \\
Loss at 16p, 16q & 6 & NA (50\%) & 24 \\
Loss at 1p, 4q, 17p & 9 & NA (>70\%) & 12 \\
Loss at 11q, 13q, 15q, 16q & 9 & NA (60-70\%) & 12 \\
Loss at 3q, 6q, 8q, 18q, 21q & 9 & NA (50-60\%) & 12 \\
Loss of 11p & 12 & $6(50 \%)$ & 10 \\
& & & \\
\hline
\end{tabular}

Abbreviations: MSI, microsatellite instability; NA, not applicable. ${ }^{\mathrm{a}} 1$ CTNNB1 mutation, 3 APC mutations.

$\mathrm{b}_{1}$ MSI-high, 2 MSI-low.

$k$-ras in the pancreas leads to development of ductal neoplasms. Hezel et $a l^{30}$ showed that loss of $L k b 1$ in murine pancreas results in disturbed acinar cell polarity, abnormal cytoskeletal organization, loss of tight junctions, and inactivation of AMPK/MARK/ SAD kinases, eventually resulting in postnatal acinar cell degeneration, acinar-to-ductal metaplasia, and pancreatic serous cystadenomas. Moreover, Habbe et $\mathrm{l}^{31}$ showed that selective mutation of $k$-ras in acinar cells results in murine pancreatic intraepithelial neoplasia of all histological grades.

In addition, alterations in genes commonly involved in pancreatic ductal adenocarcinoma carcinogenesis, including TP53, KRAS, and SMAD4 were investigated. Our results confirm previous observations that these genes do not have a major role in acinar cell carcinoma. One acinar cell carcinoma showed a TP53 mutation and one case showed loss of SMAD4 protein expression, which has not been reported previously (Table 3 ). Interestingly, one acinar cell carcinoma showed TP53-independent expression of CDKN1A, a cyclin-dependent kinase inhibitor also known as WAF1/CIP1. Although CDKN1A has not been investigated in acinar cell carcinoma previously, this phenomenon has been described in pancreatic ductal adenocarcinoma. ${ }^{32}$

Alterations in the APC/CTNNB1 pathway have been reported in 4 of $17(23.5 \%)$ acinar cell carcinoma cases. ${ }^{10}$ In our series, one patient demonstrated nuclear expression of CTNNB1 $(20 \%)$ indicating activated Wnt signaling. Although we did not find aberrant expression of mismatch repair proteins or microsatellite instability, others have reported microsatellite instability in a minority $(23 \%)$ of acinar cell carcinomas. ${ }^{10}$

Previous studies have shown a relatively high degree of genomic copy number variations in acinar cell carcinoma. ${ }^{12,24}$ Allelic loss has been reported in $>60 \%$ of acinar cell carcinomas of chromosome 
arms 1p, 4q, 11q, 13q, 15q, 16p, 16q and 17p and gain at arms 1q, $12 \mathrm{p}$ and Xq. ${ }^{12,24}$ Using MLPA, we identified gains of GATA5 at 20q13.3 (5/5 cases, $100 \%$ ), $L K B 1$ at $19 \mathrm{p} 13.3$ (4/5 cases, $80 \%$ ), and at $13 \mathrm{q}$ (BRCA2 and RB1 locus) in one case (20\%). As these are all tumor suppressor genes, amplifications of these genes most likely reflects the genomic instability in these tumors instead of being events that drive tumorigenesis, and are therefore regarded as an epiphenomenon. In addition, LOH at D17S250 was found in two cases and LOH at D5S346 in one case.

CpG island promoter methylation has not been studied in pancreatic acinar cell carcinoma previously. We identified hypermethylation of one or more tumor suppressor genes in 4/5 (80\%) of cases (Table 1). One of these genes (THBS1), methylated in one acinar cell carcinoma, has previously been described to be methylated in pancreatic ductal adenocarcinomas $(7 \%)$ and intraductal papillairy mucinous neoplasms $(12 \%) .^{33}$

To conclude, our results do not point to a role for $L K B 1$ in acinar cell carcinoma pathogenesis. Although other genes involved in mTOR signaling, such as VHL, PTEN, TSC2, and PIK3CA, may be mutated, currently there seems no rationale for treatment of sporadic acinar cell carcinoma with mTOR inhibitors. We did not find specific molecular alterations in acinar cell carcinoma, but copy number variations and hypermethylation are frequently encountered. Moreover, we confirm that molecular pathways involved in acinar cell carcinoma-tumorigenesis differ from those involved in ductal pancreatic neoplasms. Our results form an incentive for more elaborate evaluation of molecular alterations in acinar cell carcinoma, which may eventually lead to development of new therapeutic targets and improved prognosis of this disease with a dismal prognosis.

\section{Acknowledgement}

We would like to thank P Drillenburg, pathologist at the Onze Lieve Vrouwe Gasthuis Amsterdam, for generously having provided one of the acinar cell carcinoma samples that was analyzed in this study.

\section{Disclosure/conflict of interest}

The authors declare no conflict of interest.

\section{References}

1 Morohoshi T, Held G, Klöppel G. Exocrine pancreatic tumours and their histological classification. A study based on 167 autopsy and 97 surgical cases. Histopathology 1983;7:645-661.

2 Hoorens A, Lemoine NR, McLellan E, et al Pancreatic acinar cell carcinoma. An analysis of cell lineage markers, p53 expression, and Ki-ras mutation. Am J Pathol 1993;143:685-698.

3 Klimstra DS, Heffess CS, Oertel JE, et al Acinar cell carcinoma of the pancreas. A clinicopathologic study of 28 cases. Am J Surg Pathol 1992;16: 815-837.

4 Luttges J, Stigge C, Pacena M, et al Rare ductal adenocarcinoma of the pancreas in patients younger than age 40 years. Cancer 2004;100:173-182.

5 Webb JN. Acinar cell neoplasms of the exocrine pancreas. J Clin Pathol 1977;30:103-112.

6 Hruban RH, Maitra A, Kern SE, et al Precursors to pancreatic cancer. Gastroenterol Clin North Am 2007;36:831-849 vi.

7 Pellegata NS, Sessa F, Renault B, et al K-ras and p53 gene mutations in pancreatic cancer: ductal and nonductal tumors progress through different genetic lesions. Cancer Res 1994;54:1556-1560.

8 Terhune PG, Heffess CS, Longnecker DS. Only wildtype c-Ki-ras codons 12, 13, and 61 in human pancreatic acinar cell carcinomas. Mol Carcinog 1994; 10:110-114.

9 Terhune PG, Memoli VA, Longnecker DS. Evaluation of p53 mutation in pancreatic acinar cell carcinomas of humans and transgenic mice. Pancreas 1998;16: 6-12.

10 Abraham SC, Wu TT, Hruban RH, et al Genetic and immunohistochemical analysis of pancreatic acinar cell carcinoma: frequent allelic loss on chromosome $11 \mathrm{p}$ and alterations in the APC/beta-catenin pathway. Am J Pathol 2002;160:953-962.

11 Moore PS, Orlandini S, Zamboni G, et al Pancreatic tumours: molecular pathways implicated in ductal cancer are involved in ampullary but not in exocrine nonductal or endocrine tumorigenesis. Br J Cancer 2001;84:253-262.

12 Rigaud G, Moore PS, Zamboni G, et al Allelotype of pancreatic acinar cell carcinoma. Int $\mathrm{J}$ Cancer 2000;88:772-777.

13 Klumpen HJ, Queiroz KC, Spek CA, et al mTOR inhibitor treatment of pancreatic cancer in a patient with Peutz-Jeghers syndrome. J Clin Oncol 2011;29:e150-e153.

14 Jansen M, Ten Klooster JP, Offerhaus GJ, et al LKB1 and AMPK family signaling: the intimate link between cell polarity and energy metabolism. Physiol Rev 2009;89:777-798.

15 Giardiello FM, Brensinger JD, Tersmette AC, et al Very high risk of cancer in familial Peutz-Jeghers syndrome. Gastroenterology 2000;119:1447-1453.

16 Giardiello FM, Welsh SB, Hamilton SR, et al Increased risk of cancer in the Peutz-Jeghers syndrome. N Engl J Med 1987;316:1511-1514.

17 Sato N, Rosty C, Jansen M, et al STK11/LKB1 PeutzJeghers gene inactivation in intraductal papillarymucinous neoplasms of the pancreas. Am J Pathol 2001;159:2017-2022.

18 Canto MI, Goggins M, Hruban RH, et al Screening for early pancreatic neoplasia in high-risk individuals: a prospective controlled study. Clin Gastroenterol Hepatol 2006;4:766-781; quiz 665.

19 Yee NS, Furth EE, Pack M. Clinicopathologic and molecular features of pancreatic adenocarcinoma associated with Peutz-Jeghers syndrome. Cancer Biol Ther 2003;2:38-47.

$20 \mathrm{Su}$ GH, Hruban RH, Bansal RK, et al Germline and somatic mutations of the STK11/LKB1 Peutz-Jeghers 
gene in pancreatic and biliary cancers. Am J Pathol 1999;154:1835-1840.

21 de Leng WW, Jansen M, Carvalho R, et al Genetic defects underlying Peutz-Jeghers syndrome (PJS) and exclusion of the polarity-associated MARK/Par1 gene family as potential PJS candidates. Clin Genet 2007;72:568-573.

22 Boland CR, Thibodeau SN, Hamilton SR, et al A National Cancer Institute Workshop on Microsatellite Instability for cancer detection and familial predisposition: development of international criteria for the determination of microsatellite instability in colorectal cancer. Cancer Res 1998;58:5248-5257.

23 Langeveld D, van Hattem WA, de Leng WW, et al SMAD4 immunohistochemistry reflects genetic status in juvenile polyposis syndrome. Clin Cancer Res 2010;16:4126-4134.

24 Taruscio D, Paradisi S, Zamboni G, et al Pancreatic acinar carcinoma shows a distinct pattern of chromosomal imbalances by comparative genomic hybridization. Genes Chromosomes Cancer 2000;28:294-299.

25 Shackelford DB, Vasquez DS, Corbeil J, et al mTOR and HIF-1alpha-mediated tumor metabolism in an LKB1 mouse model of Peutz-Jeghers syndrome. Proc Natl Acad Sci USA 2009;106:11137-11142.

26 Robinson J, Lai C, Martin A, et al Oral rapamycin reduces tumour burden and vascularization in Lkb1 (+/-) mice. J Pathol 2009;219:35-40.
27 Wei C, Amos CI, Zhang N, et al Chemopreventive efficacy of rapamycin on Peutz-Jeghers syndrome in a mouse model. Cancer Lett 2009;277:149-154.

28 Zhang N, Lyons S, Lim E, et al A spontaneous acinar cell carcinoma model for monitoring progression of pancreatic lesions and response to treatment through noninvasive bioluminescence imaging. Clin Cancer Res 2009;15:4915-4924.

29 Jiao Y, Shi C, Edil BH, et al DAXX/ATRX, MEN1, and mTOR pathway genes are frequently altered in pancreatic neuroendocrine tumors. Science 2011;331:1199-1203.

30 Hezel AF, Gurumurthy S, Granot Z, et al Pancreatic LKB1 deletion leads to acinar polarity defects and cystic neoplasms. Mol Cell Biol 2008;28:2414-2425.

31 Habbe N, Shi G, Meguid RA, et al Spontaneous induction of murine pancreatic intraepithelial neoplasia (mPanIN) by acinar cell targeting of oncogenic Kras in adult mice. Proc Natl Acad Sci USA 2008;105: 18913-18918.

32 DiGiuseppe JA, Redston MS, Yeo CJ, et al p53independent expression of the cyclin-dependent kinase inhibitor p21 in pancreatic carcinoma. Am J Pathol 1995;147:884-888.

33 Sato N, Ueki T, Fukushima N, et al Aberrant methylation of $\mathrm{CpG}$ islands in intraductal papillary mucinous neoplasms of the pancreas. Gastroenterology 2002;123:365-372. 